$\begin{array}{ll}\text { Published in Crystal Growth \& Design 14 (2014) 2479 } \\ \text { doi: } 10.1021 / \text { cg500190h } & \text { http://pubs.acs.org/ }\end{array}$

\title{
Nano-scaled M-MOF-74 materials prepared at room temperature
} Manuel Díaz-García ${ }^{a}$ Álvaro Mayoral, ${ }^{b}$ Isabel Díaz ${ }^{a}$ and Manuel Sánchez-Sánchez ${ }^{a, *}$

${ }^{a}$ Instituto de Catálisis y Petroleoquímica, ICP-CSIC, C/ Marie Curie 2, 28049 Madrid, Spain. ${ }^{\mathrm{b}}$ Laboratorio de Microscopías Avanzadas (LMA), Instituto de Nanociencia de Aragon (INA), Universidad de Zaragoza, Mariano Esquillor, Edificio I+D, 50018, Zaragoza, Spain.

ABSTRACT. This paper describes the preparation and characterization of nano-scaled M-MOF74/CPO-27-M ( $\mathrm{M}=\mathrm{Mg}, \mathrm{Mn}, \mathrm{Co}, \mathrm{Ni}$ and $\mathrm{Zn}$ ) materials at room temperature. Some of the soformed crystals are the smallest ones of any MOF material (and, to the best of our knowledge, of any microporous material) ever reported. They are in the limit of being able to diffract, particularly these forming the Co- and Ni-MOF-74 samples. Consequently, unequivocal identification as crystalline MOF-74 phase was deduced by combining other characterization techniques rather than powder X-ray diffraction. These small crystals are unstable as isolated ones, so they form steady and robust aggregates, whose mechanical properties strongly depend on the crystal size. The particles that results from the 'fusion' of nanocrystals smaller than $10 \mathrm{~nm}$ (more properly denoted as nanodomains) could not be disaggregated by conventional ultrasonic and graining techniques. On the contrary, agglomerates of crystals larger than $10 \mathrm{~nm}$ are dissociable in discrete crystals. It allows characterizing Zn-MOF-74 nanocrystals by advanced electron microscopy methods. Cs-corrected STEM provided, for the first time, 'quasi' atomic resolution images of MOFs, which are especially unstable under electronic radiation. The magnitude of the crystal size of M-MOF-74 is tentatively associated to the solubility of the metal source.
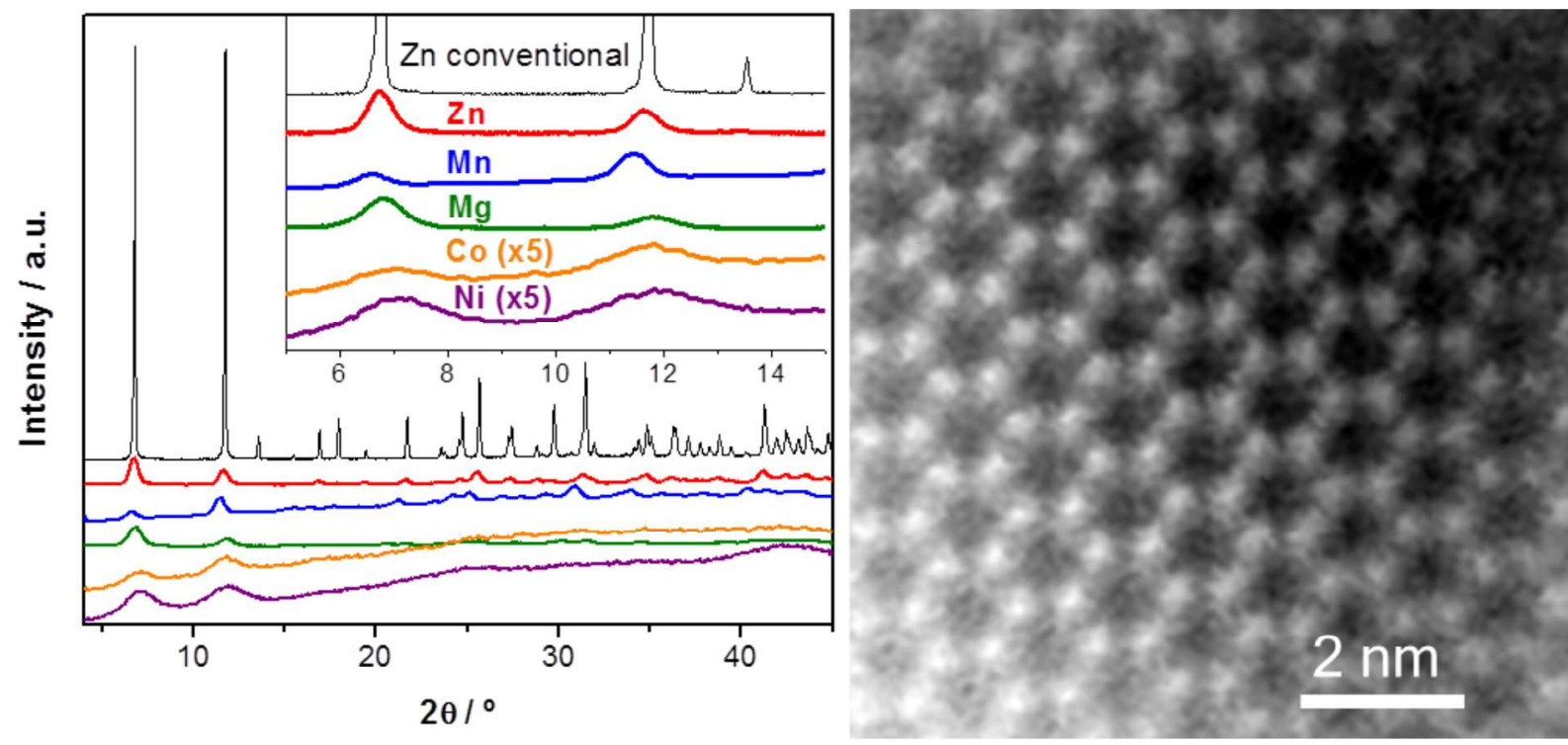

Dr. Manuel Sánchez-Sánchez Instituto de Catálisis y Petroleoquímica, ICP-CSIC

C/ Marie Curie, 2, 28049 Madrid, Spain

Tel.: +34-915854795; Fax: +34-915854760

E-mail: manuel.sanchez@icp.csic.es 


\title{
Nano-scaled M-MOF-74 materials prepared at room
}

\section{temperature}

\author{
Manuel Díaz-García, ${ }^{a}$ Álvaro Mayoral, ${ }^{b}$ Isabel Díaz ${ }^{a}$ and Manuel Sánchez-Sánchez ${ }^{a,{ }^{*}}$ \\ a Instituto de Catálisis y Petroleoquímica, ICP-CSIC, C/ Marie Curie 2, 28049 Madrid, Spain. \\ ${ }^{\mathrm{b}}$ Laboratorio de Microscopías Avanzadas (LMA), Instituto de Nanociencia de Aragon (INA), \\ Universidad de Zaragoza, Mariano Esquillor, Edificio I+D, 50018, Zaragoza, Spain. \\ *manuel.sanchez@icp.csic.es
}

\begin{abstract}
This paper describes the preparation and characterization of nano-scaled M-MOF74/CPO-27-M (M = Mg, Mn, Co, Ni and Zn) materials at room temperature. Some of the soformed crystals are the smallest ones of any MOF material (and, to the best of our knowledge, of any microporous material) ever reported. They are in the limit of being able to diffract, particularly these forming the Co- and Ni-MOF-74 samples. Consequently, unequivocal identification as crystalline MOF-74 phase was deduced by combining other characterization techniques rather than powder X-ray diffraction. These small crystals are unstable as isolated ones, so they form steady and robust aggregates, whose mechanical properties strongly depend on the crystal size. The particles that results from the 'fusion' of nanocrystals smaller than $10 \mathrm{~nm}$ (more properly denoted as nanodomains) could not be disaggregated by conventional ultrasonic and graining techniques. On the contrary, agglomerates of crystals larger than $10 \mathrm{~nm}$ are dissociable in discrete crystals. It allows characterizing Zn-MOF-74 nanocrystals by advanced electron microscopy methods. Cs-corrected STEM provided, for the first time, 'quasi' atomic resolution images of MOFs, which are especially unstable under electronic radiation. The
\end{abstract}


magnitude of the crystal size of M-MOF-74 is tentatively associated to the solubility of the metal source.

KEYWORDS. MOF-74, CPO-27, nanocrystals, nanodomains, Cs-corrected STEM, Scherrer, 'quasi' atomic resolution. 


\section{INTRODUCTION}

The discovery of permanent porosity in Metal-Organic Framework (MOF) materials ${ }^{1}$ has revolutionized the research field of microporous materials in the last fifteen years ${ }^{2,}{ }^{3}$. Accordingly, the already-established applications of microporous materials have been consolidated and intensified, whereas some other novel applications have been either described or postulated as likely ${ }^{2-4}$. The extraordinary topological and compositional versatility of MOFs ${ }^{2}$, 3,5 , unbeaten by any other family of materials, is behind their potentiality in industrial applications.

Some of these applications require (or at least could work more adequately with) the use of materials being formed by nano-sized crystals ${ }^{6-12}$. Thus, the application of MOFs as heterogeneous catalysts must face the deactivation of the catalytic centers, either by centers poisoning or by simple pore blockage. Reaching the nano-scaled crystal size of microporous catalysts would reduce the kinetics of the pore blockage ${ }^{13}$, and the regeneration process of the poisoned centers could be carried out quicker and/or under softer conditions, more compatible with the relatively low thermal stability of MOF materials. In this sense, the catalytic activity of a particular MOF material has enhanced by decreasing their crystal size ${ }^{14}$. As a second illustrative example of the convenience of obtaining nano-MOFs, the preparation, mechanical properties and efficiency in gas separation of the mixed matrix membranes formed by a polymer matrix and a nanoporous filler has been reported to improve with the crystal size reduction of the filler $^{15}$, whereas the substitution of zeolites by MOFs as fillers gives some important affinity advantages with the polymer ${ }^{16}$.

Apart from the solvothermal method, almost exclusively applied to the synthesis of allinorganic microporous materials ${ }^{17}$, a series of synthesis methods has been developed for 
preparing $\mathrm{MOFs}^{18}$, quite often precisely designed to reduce the crystal size. Thus, synthesis methods assisted by mechanical mills ${ }^{19}$, by sonochemical equipments ${ }^{20}$ or by microwave radiation $^{21}$ (the latter being also of solvothermal nature) have been indeed reported as successful in this aspect. The synthesis method carried out at room temperature is even more attractive from both industrial and environmental points of view, due to the unneeded input of any energy source in the MOF formation processes. Although the quality of the MOF materials prepared at room temperature has been questioned and demonstrated to be poor in some particular cases ${ }^{22,23}$, probably because their formation was forced by, for instance, abrupt $\mathrm{pH}$ changes, in some other cases they have reached equal qualities to those materials formed by large crystals and prepared under solvothermal conditions ${ }^{24}$.

The isostructural materials known as M-MOF- $74^{25}, \mathrm{CPO}-27-\mathrm{M}^{26}$ or $\mathrm{M}(\mathrm{dhtp})_{2}{ }^{27}$ have awakened notable attention due to the presence of unsaturated metal centers in the evacuated material, their versatility of being prepared with different divalent ions ${ }^{27,} 28$ or mixture of them at any extension $^{29}$, and/or their stability under ambient conditions and in presence of water ${ }^{30}$. These materials have distinguished behavior in adsorption of gases of high environmental and/or energetic interest even amongst the rest of the open-metal-sites containing MOF materials. Thus, CPO-27- $\mathrm{Mg}^{27}, 31,32$ is one of the materials showing higher capacity and selectivity to adsorb $\mathrm{CO}_{2}{ }^{33}$ and gives one of the highest isosteric heats for $\mathrm{H}_{2}$ physisorption ${ }^{34,35}$. Additionally, the interest in this topology has recently increased due to the discover of the so-called series of IRMOF-74, formed by materials isoreticular to MOF-74 by changing the length of the linker but maintaining their terminal functional groups, their denticity and therefore their coordination to the metal cluster. A material belonging to that series reaches a pore diameter as large as $85 \times 98$ $\AA^{36}$. Furthermore, the definitive reason of basing this study on the M-MOF-74 materials lie on 
the already-described preparation of a high-quality Zn-MOF-74 material at room temperature by a precipitation process rather than by a crystallization one $\mathrm{e}^{24}$. It concedes us certain control on the crystal size through parameters such as acid character of the starting mixture or solubility of the reactants sources.

This paper describes the preparation of a series of MOF materials formed by the smallest nanocrystals / nanodomains ever reported. They are so small that are below the detection limit of conventional laboratory X-ray diffraction (XRD) equipment. In contrast, $\mathrm{C}_{\mathrm{s}}$ HAADF-STEM images of the nano-sized Zn-MOF-74 samples showed unprecedented high-resolution in MOFs, so that quasi atomic resolution was reached. The results from an extensive characterization have made clear that not only the absolute crystal/domain size but also the way in which they are aggregated or fused, are crucial for specific applications.

\section{EXPERIMENTAL SECTION}

2.1. Synthesis. The synthesis method for preparing nano-scaled $\mathrm{Zn}, \mathrm{Ni}, \mathrm{Co}, \mathrm{Mn}$ and $\mathrm{Mg}-\mathrm{MOF}-$ 74 materials is a modification of the only one reporting the synthesis of a MOF-74 materials (the Zn-based one) at room temperature.[16] A clear yellow solution of $0.200 \mathrm{~g}(1.0 \mathrm{mmol})$ of 2,5dihydroxyterphthalic acid (dhtp) in $5.0 \mathrm{~g}$ of DMF was added dropwise over other clear solution of $2.6 \mathrm{mmol}$ of the corresponding divalente metal acetate tetrahydrate (except for $\mathrm{Zn}$ acetate that was dihydrate), immediately leading to a precipitate, which after a few minutes contains MOF74 as the only crystalline phase. The optimized reaction time was of 20 hours, after which the solid was recovered by centrifugation and washed first with $20 \mathrm{~mL}$ of DMF and then two more times with $20 \mathrm{~mL}$ of methanol. The solid was kept immersed in methanol for 6 days, changing that solvent for the same amount of fresh one three times. 
2.3. Characterization techniques. X-ray diffraction patterns were collected with a Philips X'PERT diffractometer having a $\mathrm{X}^{\prime}$ Celerator detector and using $\mathrm{Cu} \mathrm{K} \alpha$ radiation. Width crystal size was estimated by applying Scherrer equation to the $110\left(2 \Theta \sim 6.8^{\circ}\right)$ and $300\left(\sim 11.6^{\circ}\right)$ reflections of the patterns registered with $2 \Theta$ steps of $0.0167^{\circ}$ and radiation time of $100 \mathrm{~s}$ per point. Nitrogen adsorption/desorption isotherms were measured at $-196{ }^{\circ} \mathrm{C}$ in a Micromeritics ASAP 2420 device. Before, the previously calcined samples were degassed at $350{ }^{\circ} \mathrm{C}$, respectively, under high vacuum for at least 18 hours. Surface areas were estimated by BET method. Micropore and external surface area was estimated from t-plot method. Pore size distributions were obtained by application of BJH method to the desorption branch of the N2 isotherms. Thermogravimetric analysis (TGA) using a Perkin-Elmer TGA7 instrument. TG analyses were carried out at a heating rate of $20^{\circ} \mathrm{C} / \mathrm{min}$ under air flow. Scanning Electron Microscopy (SEM) studies were carried out in a Ultra-high Resolution FEI-NOVA NanoSEM 230 FESEM instrument. Transmission Electron Microscopy (TEM) images were obtained in a JEOL 2000FX microscope operated with an accelerating voltage of $200 \mathrm{kV}$. Aberration (Cs) corrected STEM was performed in a FEI TITAN X-FEG (60-300) transmission electron microscope equipped with a monochromator, a spherical aberration CEOS corrector for the electron probe and a Gatan Tirdiem energy filter (GIF). For the current experiments the voltage was set to $300 \mathrm{kV}$ yielding a probe size of $0.8 \AA$ while the beam current was set $<1.65 \times 10-10$ A using a convergence semiangle of $17 \mathrm{mrad}$.

\section{RESULTS}

3.1. X-ray diffraction. Powder XRD patterns of the M-MOF-74 (M = Mg, Mn, Co, Ni or Zn) materials prepared at room temperature $(293 \mathrm{~K})$ for 24 hours under stirring are shown in Figure 
1. For comparison purposes, the XRD pattern of a Zn-MOF-74 material prepared under conventional solvothermal conditions ${ }^{29}$ in our laboratory is also included in that Figure. The most evident feature of Figure 1 is a major broadening of the peaks found in the XRD patterns of the samples prepared at room temperature with respect to those of the conventional Zn-MOF-74. The XRD patterns of Figure 1 have been ordered according to the peak broadening, from the widest one (bottom) to narrowest one (top). In spite of the remarkable peak broadening, the identification of the MOF-74 phase is more or less evident in the case of the samples prepared with $\mathrm{Mg}, \mathrm{Mn}$ or $\mathrm{Zn}$. However, peak broadening becomes so large in the case of the Co and $\mathrm{Ni}$ samples that just a couple of shoulders, those found at the lowest $2 \Theta$ position and corresponding to the Miller index 110 and 300, are intuited, questioning that MOF-74 is the present phase and even the crystalline nature of the sample. The significant background of the $\mathrm{Mn}, \mathrm{Ni}$ and $\mathrm{Co}-$ based samples is mainly due to the fluorescence provoked by these metal elements when the Xray radiation source is $\mathrm{Cu} \mathrm{K \alpha}$ (see Figure S1-S3 and S5 in Supporting Information) although the contribution of some amorphous phase cannot a priori be discarded.

Therefore, the identification of the phase MOF-74 through powder X-ray diffraction is far from being enough, at least for the $\mathrm{Ni}$ and Co-based samples, and accordingly extra and unequivocal indications from some other different characterization techniques are required. In this sense, a variety of strong evidences (see below) are presented along this article certifying that all five samples whose XRD patterns are compiled in Figure 1 are crystalline and possess a MOF-74 structure. Taking this into account, it is appropriate to estimate the crystal size of the samples through X-ray diffraction peak broadenings. Although it has been made clear the convenience of using other methods different to that based on Scherrer equation, particularly in microporous materials ${ }^{37,} 38$, the exclusive detection of only two peaks in some of the 


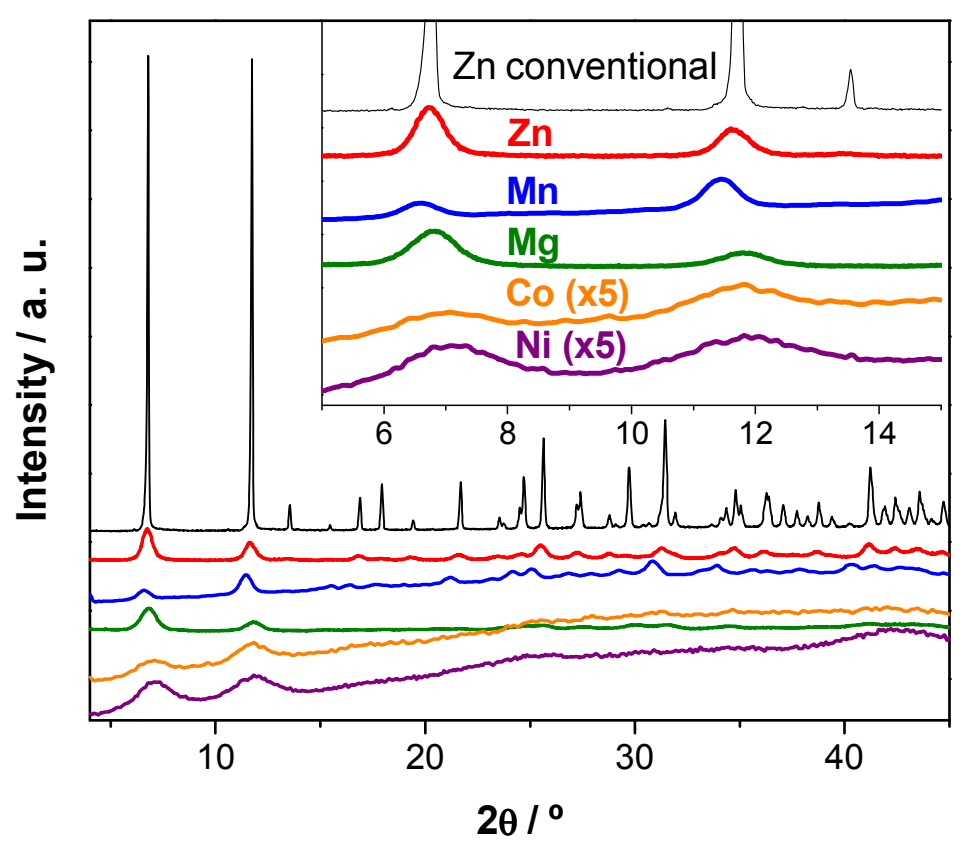

Figure 1. Powder X-ray diffraction patterns of the M-MOF-74 samples prepared at room temperature (from bottom to top, $\mathrm{M}$ is $\mathrm{Ni}, \mathrm{Co}, \mathrm{Mg}, \mathrm{Mn}, \mathrm{Zn}$ ) and a $\mathrm{Zn}-\mathrm{MOF}-74$ material (top) prepared by conventional method. The intensity of the patterns of $\mathrm{Ni}$ and Co samples is multiplied by 5 to facilitate the comparison. The enlargement shows the $2 \Theta$ region of $5-15^{\circ}$ of the same patterns.

diffractograms of Figure 1 force us to use such equation. In general, it is accepted that Scherrer equation is more precise for very small crystals, that is, for relatively large broadenings. However, in the case of $\mathrm{Co}$ and $\mathrm{Ni}$ samples the broadening is so remarkable that may compromise its own resolution, as the diffraction band is somehow confused with the background. In order to gain precision, the Scherrer equation was applied to XRD patterns of these two samples acquired for very much longer times each point and maintaining the number of points. Figure S4 from Supplementary information contains some of the so-acquired XRD patterns compared with that of the same samples shown in Figure 1, and Table S1 shows the detailed data of the crystal size estimations by applying Scherrer equation. The instrumental broadening was taken from the same peaks of an equally-registered XRD pattern of a Zn-MOF74 material prepared under conventional conditions ${ }^{29}$ and therefore formed by relative large crystals. The resulting averaged crystal size from that estimations were of 16.6, 13.6, 9.4, 5.1 and $2.8 \mathrm{~nm}$ for the M-MOF-74 samples with M being Zn, Mn, Mg, Co and Ni, respectively. 
3.2. FT-IR spectroscopy. Probably, the most extended use of PXRD technique is as fingerprint to identify /refute a / some already-known and solved structure(s). Unfortunately, Figure 1 have made clear that phase identification is not possible in the case of $\mathrm{Co}$ and $\mathrm{Ni}$ samples, and it is even uncertain for the rest of PXRD patterns due to their considerable peak broadening. However, some other techniques apart from PXRD can also work based on a homologue fingerprint strategy. This is the case of infrared spectroscopy, which is not as sensitive as PXRD to the framework topology but it is quite sensitive to the conformational and/or local environment of organic molecules in the so-called fingerprint region (ca. 500-1700 $\mathrm{cm}^{-1}$ ). Accordingly, deprotonation of the carboxylate groups and their consequent bond to the divalent metals must be evidenced by this spectroscopy. Figure 2 shows the IR fingerprint region of the spectra registered for the five nanocrystalline samples, for the conventional Zn-MOF-74 and for the organic linker. As expected, IR spectra of the linker and the conventional Zn-MOF74 are markedly different. Likewise, there is a very good agreement between band position and relative intensity between IR spectrum of the nanocrystalline Zn-MOF-74 and that of the conventional Zn-MOF-74. Hence, the match of both FTIR spectra with the spectrum of the linker is quite poor. The most evident difference between the spectra of nano-sized and conventional $\mathrm{Zn}$ samples is the lower resolution of the bands in the former, which is attributed to the 'nano' nature of their crystals. In addition, the band broadening increases from top to bottom, Figure 2, as a consequence of the decrease of the crystal size of the samples. Obviously, the influence of the nanocrystalline character on spectroscopy does not reach the magnitude of that on the X-ray diffraction broadening, since the latter is a technique of much longer range. However, the simple fact of provoking so perceptible broadening in IR bands gives an idea of extraordinarily small crystal size ${ }^{39}$. The IR spectrum of the Mn-MOF-74 sample contains some singularities (extra 


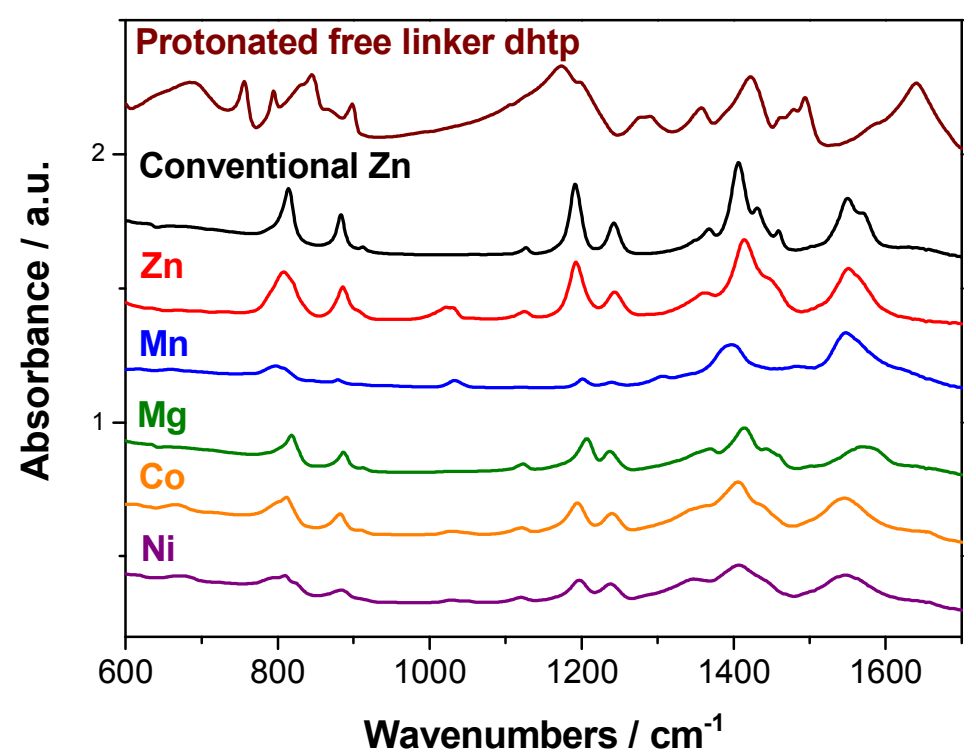

Figure 2. FTIR-ATR spectra of the M-MOF-74 samples prepared at room temperature (from bottom to top, $\mathrm{M}$ is $\mathrm{Ni}, \mathrm{Co}, \mathrm{Mg}, \mathrm{Mn}, \mathrm{Zn}$ ), a $\mathrm{Zn}-\mathrm{MOF}-74$ material prepared by conventional method and the protonated free linker dhtp (top).

bands, different relative intensity, etc.) in comparison with the other spectra of the nanocrystalline samples. Nevertheless, even this spectrum mainly follows the main IR pattern of the series and, in any case, it is decidedly different to the IR spectrum of the linker. Therefore, IR studies confirm that all these MOF samples prepared at room temperature are crystalline, suggest that they are indeed formed by crystals of nano-scaled size and that they most likely have a MOF-74 topology, including those more doubtful cases because of its negligible / limited capacity of diffracting X-rays.

3.3. Thermogravimetric analyses. Figure 3 shows the thermogravimetric profiles of the free linker used in the synthesis of MOF-74 materials, the as-synthesized nanocrystalline M-MOF-74 materials and the conventional Zn-MOF-74 after being washed several times with methanol. In good agreement with the literature ${ }^{40}$, the linker decomposition temperature increases in the order: $\operatorname{Mn}\left(307^{\circ} \mathrm{C}\right)>\operatorname{Co}\left(331{ }^{\circ} \mathrm{C}\right)>\operatorname{Ni}\left(346^{\circ} \mathrm{C}\right)>\operatorname{linker} \operatorname{dhtp}\left(357^{\circ} \mathrm{C}\right)>\operatorname{Zn}\left(397^{\circ} \mathrm{C}\right)>$ Conventional $\mathrm{Zn}\left(416^{\circ} \mathrm{C}\right)>>\mathrm{Mg}\left(526^{\circ} \mathrm{C}\right)$. The temperatures given in brackets are the maximum of the 
derivative peak, and they are different from the reported data presumably because of the different heating program and atmosphere conditions.

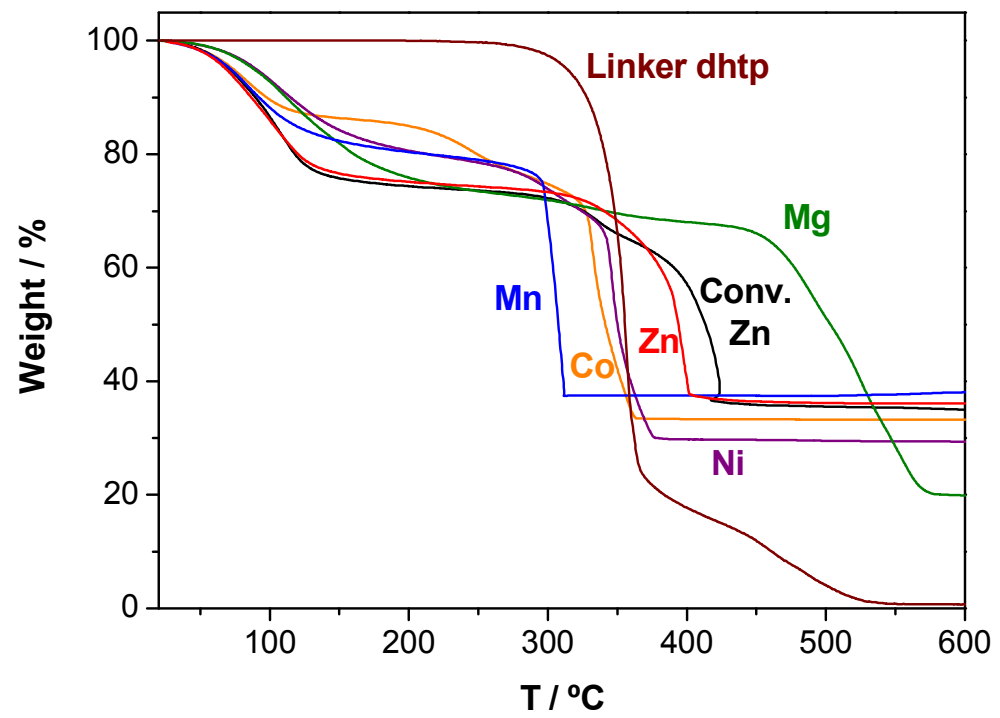

Figure 3. TGA curves of the M-MOF-74 samples prepared at room temperature, a Zn-MOF-74 material prepared by conventional method and the linker dhtp.

Figures S11 and S12 of Supporting Information compare the TGA plots of the Zn- and Cobased MOF-74 samples, both prepared by two methods: the conventional one and the roomtemperature one reported in this work. The difference of $\sim 11{ }^{\circ} \mathrm{C}$ between the detected linker decomposition temperatures of nano- and micrometer-crystalline Zn-based MOF-74 samples should be attributed to the well-known influence of crystal size on the dynamic thermogravimetric technique, so that the weight loss of any chemical species is generally found at lower temperatures when the sample is formed by smaller crystals. Therefore, that small difference provided by TGA measurements supports the same phase nature of both Zn-based MOF-74 samples with just some differences in crystal size. However, the order of the linker decomposition is the opposite when micrometer- and nano-sized Co-based MOF-74 samples are compared (maximum of decomposition temperature of 301 and $331{ }^{\circ} \mathrm{C}$, respectively). This fact, together with the SEM characterization of the 'nano' Co-MOF-74 sample (Figures 6, S21-22), 
led us to consider that this sample is formed by large particles of fused nano-sized crystals, that is, those particles are formed by Co-MOF-74 nanodomains rather by agglomerates or aggregates of 'real' discrete nanocrystals. The larger crystals of the residual $\mathrm{Co}_{3} \mathrm{O}_{4}$ formed from the decomposition of RT-prepared MOF-74 in comparison with the same oxide phase formed from the same treatment of the conventional Co-MOF-74 (Figure S8) also supports that interpretation.

On the other hand, the ratio between the weight loss of linker and the residual inorganic mass after decomposition is practically the same in thermograms of micrometer- and nano-sized samples of the same metal (Table S2: such ratio is of 1.04 for both Zn-samples, and it is of 1.54 and 1.58 for conventional and nano-sized Co-MOF-74 samples, respectively). Moreover, the TGA plots of the nanocrystalline M-MOF-74 properly matches with those published elsewhere for their homologue M-MOF-74 materials prepared under conventional conditions, ${ }^{40}$ in terms of magnitude of the weight losses, order of thermal stability, and identification of the residual phases after linker decomposition. Therefore, TGA provides important features, once again suggesting that all our samples are indeed nanocrystalline M-MOF-74.

3.4. $\mathbf{N}_{2}$ adsorption/desorption isotherms. $\mathrm{N}_{2}$ adsorption/desorption isotherms of the series of M-MOF-74 samples, registered at $-196{ }^{\circ} \mathrm{C}$, are shown in Figure 4. Some textural values estimated from those isotherms are compiled in Table 1. In good agreement with literature, the isotherm of the conventional Zn-MOF-74 is basically of type I according to the IUPAC classification of isotherms shapes ${ }^{41}$, indicating that its porosity is almost entirely due to its micropores (Table 1). In contrast, certain $\mathrm{N}_{2}$ adsorption in $\mathrm{p} / \mathrm{p}_{0}$ mesopore and macropore region and the presence of more or less marked hysteresis loop were found in every isotherm of the nanocrystalline M-MOF-74 samples. The magnitude and the type of that porosity strongly depend on the nature of the divalent metal. Figure 5 presents excellent examples of every type of 


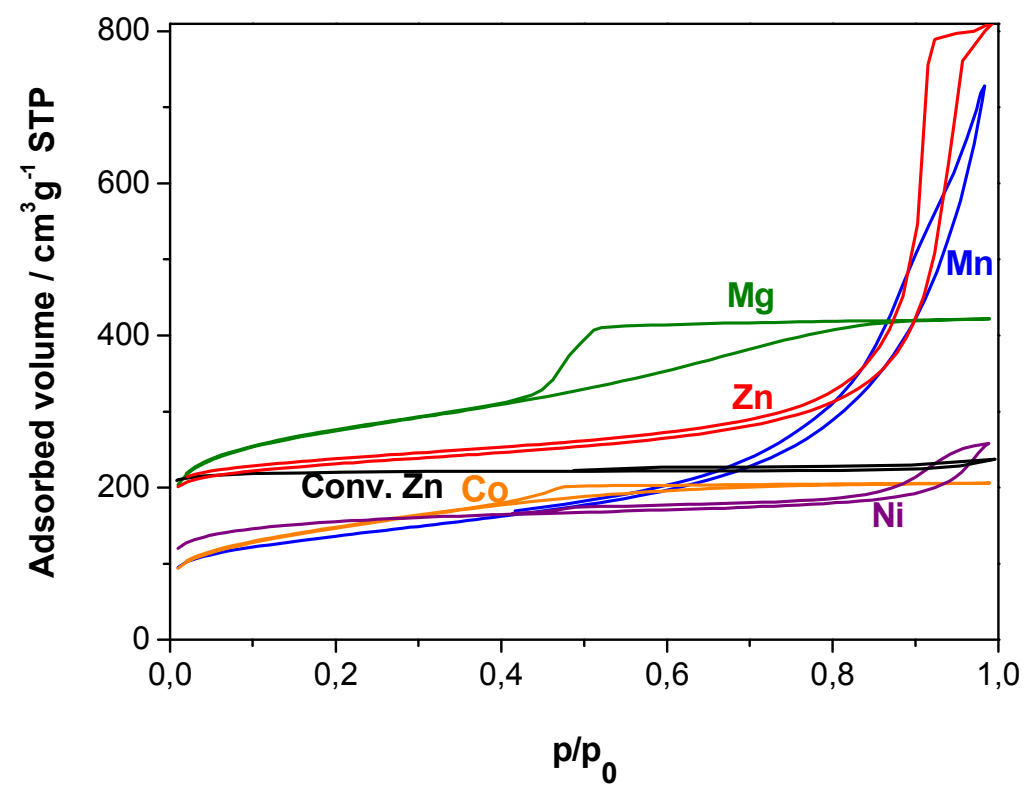

Figure 4. $\mathrm{N}_{2}$ adsorption/desorption isotherms of the M-MOF-74 samples prepared at room temperature, and a Zn-MOF-74 material prepared by conventional method.

hysteresis loops classified by IUPAC ${ }^{41}$. Thus, the nanocrystalline Zn-based sample, which contains lower microporosity than its micrometer-crystalline homologue, is able to adsorb very much higher amount of $\mathrm{N}_{2}$, due to the presence of well-defined mesopores and/or the increment of external surface area (Table 1), presumably as a consequence of the nano-sized of its crystals. Both the abrupt increase of adsorbed $\mathrm{N}_{2}$ at a particular pressure (at $\mathrm{p} / \mathrm{p}_{0}$ of $c a$. 0.9 ) of the mesopore region and the presence of well-defined and symmetric hysteresis loop, suggest a very narrow distribution of crystals size. Indeed, the classic type $\mathrm{H} 1$ of its hysteresis loop is characteristic of agglomerates (rather than aggregates, understanding agglomerate as an assemblage of particles rigidly joined together ${ }^{41}$ ) of quite uniform crystals in fairly regular array, and hence to have narrow distributions of pore size ${ }^{41}$. The slight deviation from the ideal shape of H1 loop could be due to the non-spherical rod-like morphology of the crystals (see microscopy section below). Remarkably, the shape of the hysteresis loop of the nanocrystalline Mn-MOF-74 sample, which is formed by the second largest crystals amongst the series of nano- 
sized M-MOF-74, only overcame by its Zn-based homologue, is similar to that of the Zn-MOF74 sample in the whole amount of $\mathrm{N}_{2}$ adsorbed in the mesopore region as well as in the magnitude and shape of the hysteresis loop. The lower microporosity found in the isotherm of the Mn-based sample was certified by the detection of some impurities by SEM studies (Figure S15). Its hysteresis loop is of type H3, not exhibiting any limiting adsorption at high $\mathrm{p} / \mathrm{p}_{0}$, as it is characteristic of crystal agglomerates (rather than aggregates, understanding agglomerate as an assemblage of particles which are loosely coherent ${ }^{41}$ ). Continuing with the order of crystal size, the Mg- and Co-based samples have similar features in hysteresis loop but distinct to these of the $\mathrm{Zn}$ - and Mn-based nanocrystalline samples. Their hysteresis loops are unquestionably of type $\mathrm{H} 2$, indicating that the mesopores are widely interconnected ${ }^{41}$. Far from being a disadvantage, that high pore interconnectivity could be an extra benefit for avoiding the diffusional problems of reactants/products within MOF-based heterogeneous catalysts. Nevertheless, based on recent studies $^{42}$, the shape of the hysteresis loop of the isotherms of the nanocrystalline $\mathrm{Mg}$ - and CoMOF-74 samples could be a simple consequence of the reduction of the mesopore size rather than any feature related to the mesopore interconnectivity. Finally, the hysteresis loop of the isotherm of the Ni-MOF-74 basically resembles that of a type H4. H4 loop associated to an isotherms of type I is simply indicative of microporosity. In fact, the almost negligible hysteresis loop of the isotherm of the micrometer-crystalline $\mathrm{Zn-MOF-74,} \mathrm{whose} \mathrm{unique} \mathrm{pores} \mathrm{must} \mathrm{be}$ micropores, is also of type H4. It is reasonable to admit that the aggregation of sub-3-nm crystals is not able to define mesopores but micropores $(<2 \mathrm{~nm})$. In this sense, the PSD maximum of the IUPAC limit between micro- and mesopore. 
Table 1. Textural values of the nanocrystalline M-MOF-74 materials estimated from the $\mathrm{N}_{2}$ adsorption/desorption presented in Figure 4.

\begin{tabular}{|c|c|c|c|c|c|c|}
\hline Sample & $\begin{array}{l}S_{\text {BET }}^{|a|} \\
\left(\mathrm{m}^{2} \mathrm{~g}^{-1}\right)\end{array}$ & $\begin{array}{l}S_{\text {microp }} \text { |b] } \\
\left(\mathrm{m}^{2} \mathrm{~g}^{-1}\right)\end{array}$ & $\begin{array}{l}S_{\text {ext }}^{|c|} \\
\left(m^{2} g^{-1}\right)\end{array}$ & $\begin{array}{l}V_{\text {pore }}{ }^{|d|} \\
\left(\mathrm{cm}^{3} \mathrm{~g}^{-1}\right)\end{array}$ & $\begin{array}{l}V_{\text {micropore }}^{\text {Ie] }} \\
\left(\mathbf{c m}^{3} \mathbf{g}^{-1}\right)\end{array}$ & $\begin{array}{l}\text { PSD max. }{ }^{[\mathrm{If}]} \\
(\mathrm{nm})\end{array}$ \\
\hline Conv. Zn & 850 & 841 & 9 & 0.34 & - & - \\
\hline $\mathrm{Zn}$ & 867 & 695 & 172 & 1.03 & 0.28 & 29.3 \\
\hline $\mathrm{Mn}$ & 491 & 301 & 190 & 1.04 & 0.08 & 21.5 \\
\hline $\mathrm{Mg}$ & 1007 & 607 & 400 & 0.65 & 0.25 & 5.2 \\
\hline $\mathrm{Co}$ & 521 & 128 & 393 & 0.32 & 0.06 & 2.4 \\
\hline $\mathrm{Ni}$ & 402 & 346 & 56 & 0.27 & 0.14 & $25.6^{[\mathrm{d}]}$ \\
\hline
\end{tabular}

[a] Total surface area estimated by BET method. [b] BET surface area of micropores. [c] BET external surface area. [d] Total pore volume. [e] Micropore volume. [f] Maximum value of the pore size distribution from adsorption branch. [g] Unlike the PSD maximum detected for the rest of the nanocrystalline M-MOF-74, this PSD maximum is not related to the nanocrystalline nature of the sample.

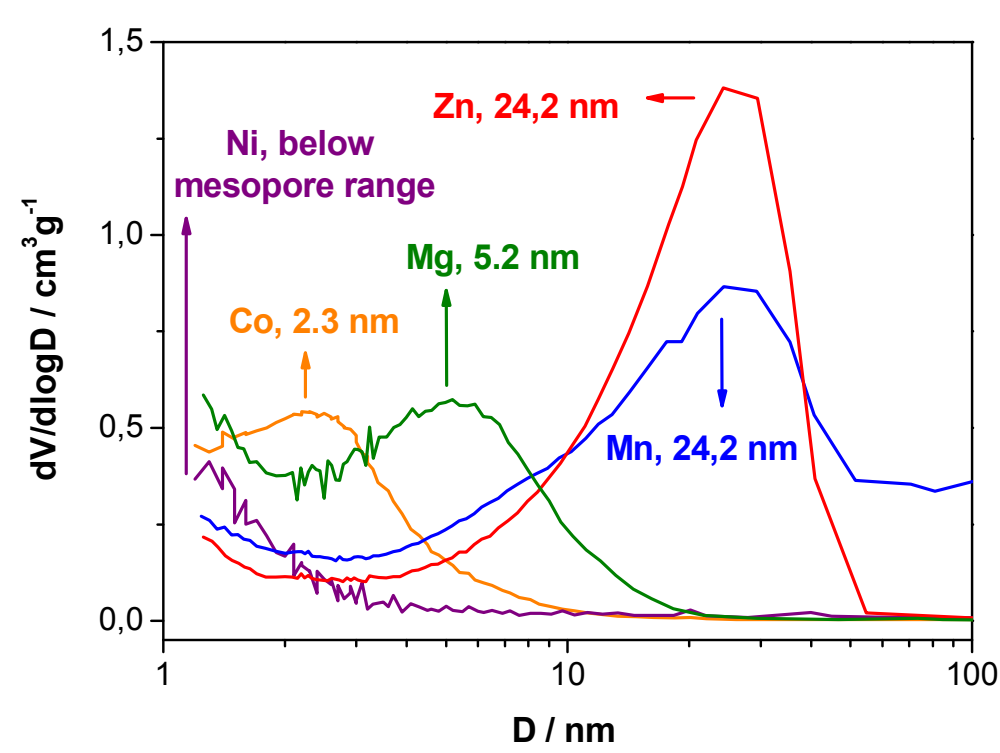

Figure 5. $\mathrm{N}_{2}$ adsorption/desorption isotherms of the M-MOF-74 samples prepared at room temperature, and a Zn-MOF-74 material prepared by conventional method.

The comparison of the textural properties of the conventional and nanocrystalline Zn-MOF-74 samples underline the similarities and the difference between both samples. Thus, the slightly higher surface area of the nanocrystalline evidences the high quality of this sample. However, its micropore surface area is significantly lower than that of its homologue formed by large crystals, which certifies their nanocrystalline nature. It is well-known that micropore surface area diminishes when crystal size decreases ${ }^{43}$ within the nano-range. Obviously, that microporosity 
loss is compensated by a notable external surface area $\left(234 \mathrm{~m}^{2} / \mathrm{g}\right)$, which is basically absent in the conventional Zn-MOF-74 $\left(9 \mathrm{~m}^{2} / \mathrm{g}\right)$. Moreover, the external surface area is presumably due to a ordered agglomeration of quite regular nano-crystals, according to its narrow pore size distribution (Figure 5) and its narrow and intense band detected by low-angle XRD (Figure S6). Although there is no a linear correlation between the crystal size of the M-MOF-74 samples and their surface area (partially because of the different atomic mass of the metals ${ }^{27}$ and the presence of impurities accompanying the Mn-MOF-74 phase (Figure S15)), there is a systematic decrease of the ratio between total and external surface area, the latter being almost two thirds of the former in the case of Co-MOF-74 sample (Table 1). Such systematic behavior seems to be violated when Ni-MOF-74 is considered. However, in that sample the crystal size are so small that the pores enclosed by them are not meso- but micropores, what could explain the substantial increase of the micropore surface area (and micropore volume) of Ni-MOF-74 with respect to that of the Co-MOF-74, which is paradoxically composed by larger crystals.

Another systematic trend with the crystal size is found in the mesopore size distribution (Table 1 and Figures 5 and S13): the smaller the crystal size, the smaller is the PSD maxima. In the case of Ni-MOF-74 samples, whose crystal domains is as small as $2.8 \mathrm{~nm}$ according to Scherrer equation, no PSD maxima of that order of magnitude is detected, probably because of the porosity generated by so small crystals is going to be within the range of micropores (pores below $2.0 \mathrm{~nm}$ ) rather than within the mesopore region.

3.5. Electron microscopy studies. In spite of the strong evidences provides by FTIR, TGA and $\mathrm{N}_{2}$ isotherms about the true nature of the nanocrystalline M-MOF-74 samples, the scarce information extracted from PXRD studies led us to make a special effort in the characterization of the samples by advanced electron microscopy techniques. Figure 6 shows the SEM 

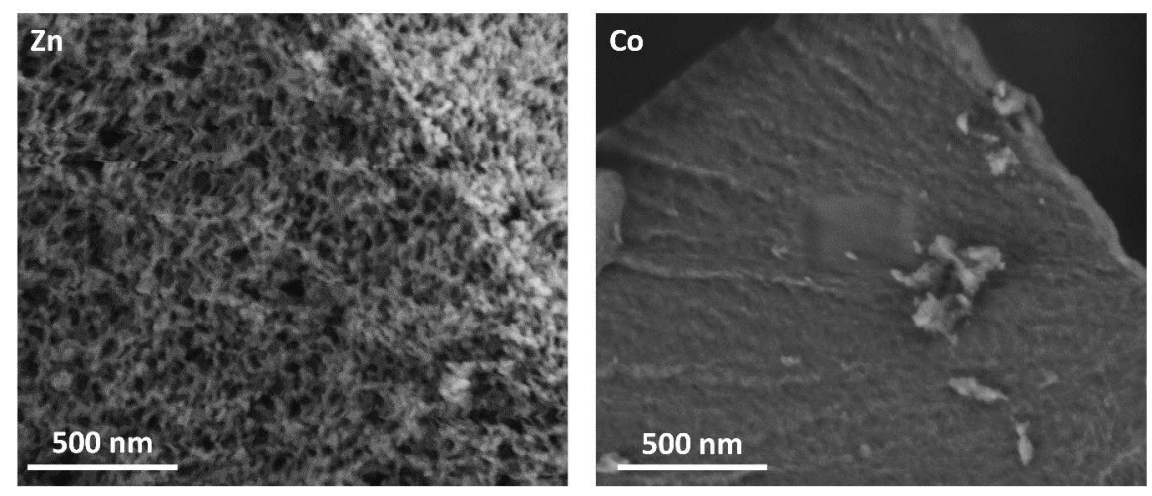

Figure 6. Highly-magnified SEM images of the as-centrifuged nanocrystalline $\mathrm{Zn}$ (left) and Co-MOF-74 (right) samples. At the centre of the latter, a previously-studied rectangular region is visible, making clear the severe damage of SEM irradiation on the MOF materials.

micrographs of the as-centrifuged $\mathrm{Co}$ and $\mathrm{Zn}$ samples, which have been selected as representative ones of being able and almost unable to diffract, respectively, according to Figure 1. $\mathrm{Zn}$ sample is composed by aggregates of nano-crystals forming a network that contains mesoporous of a relatively uniform size, in good agreement with the mesopore size distribution given by $\mathrm{N}_{2}$ adsorption-desorption isotherms. However, in the Co sample, it was not possible to discern discrete crystal units. Instead, micrometer-sized agglomerates of 'fused' crystals were observed. The as-centrifuged agglomerates / aggregates were tried to be separated in their isolated crystals by conventional methodologies based on grinding and ultrasonic treatments. Separation was achieved for the Zn aggregates but not for the agglomerates of Co or Ni samples. Supplementary information contains some extra SEM/TEM/STEM images of these and other ascentrifuged M-M-MOF-74 samples (Figure S15-S23) and DLS of the colloidal as-prepared (Figure S24), diluted and sonicated suspension before centrifugation, both supporting this interpretation. 
Figure 7a shows a conventional TEM image of the nano-sized Zn-MOF-74, with high homogeneity in shape and size of the elongated crystals. Although the crystal separation was complete for some aggregates, some nano-crystals still were linked each other at some extension. In this sense, it is not surprising that the disaggregation was unsuccessful in the case of Co or $\mathrm{Ni}$ samples (Figures 7 and S21-S23). The morphology of nano-sized crystals of Zn-MOF-74 resembles that found for conventional micrometer-sized Zn-MOF-74 materials. ${ }^{29}$ The estimation of the average crystal size was of $\approx 15 \times 50 \mathrm{~nm}$, in good agreement with the Scherrer equation valuation from XRD peak broadenings (16.6 nm, Tables S1-S2), considering that only the width (and not the length) of the elongated crystals was estimated by Scherrer equation due to the studied reflections.
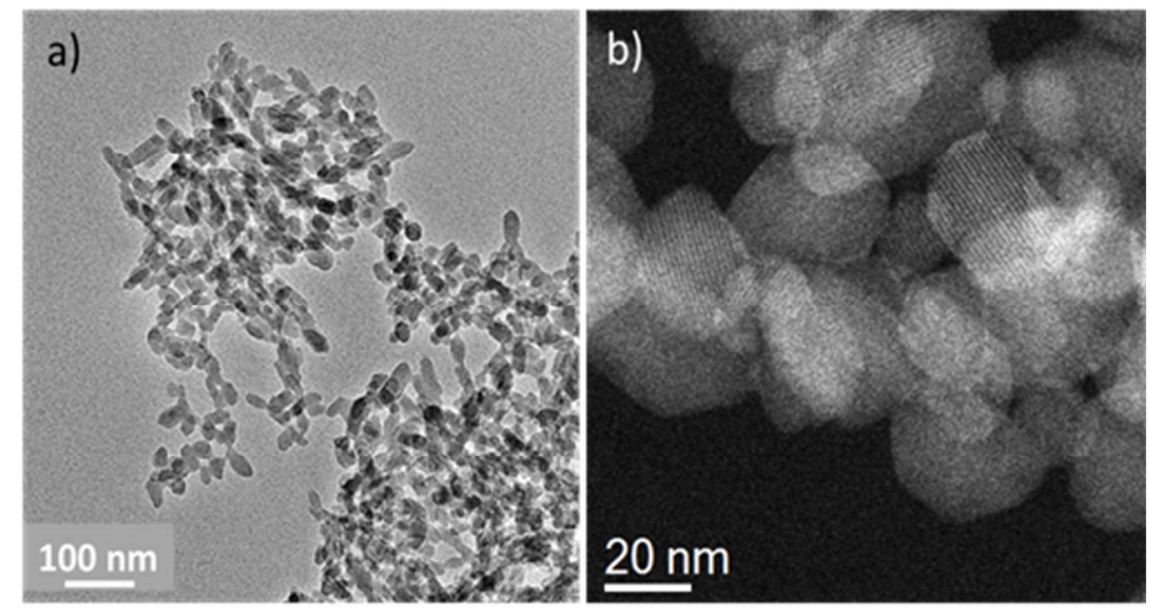

Figure 7. Micrographs of Zn-MOF-74 taken by a) conventional TEM image and b) $\mathrm{C}_{\mathrm{s}}$-corrected STEMHAADF showing the MOF channels

With the aim of gaining structural information of the crystals, spherical aberration $\left(\mathrm{C}_{\mathrm{s}}\right)$ corrected STEM-HAADF analysis was performed on the Zn-MOF sample (Figure 7b). In this mode the beam which is converged into a very fine spot, is tracked over the area of interest while the rest of the crystal remains undamaged. With this method and by taking a very careful control of the beam current atomic resolution data has been obtained on highly beam sensitive 

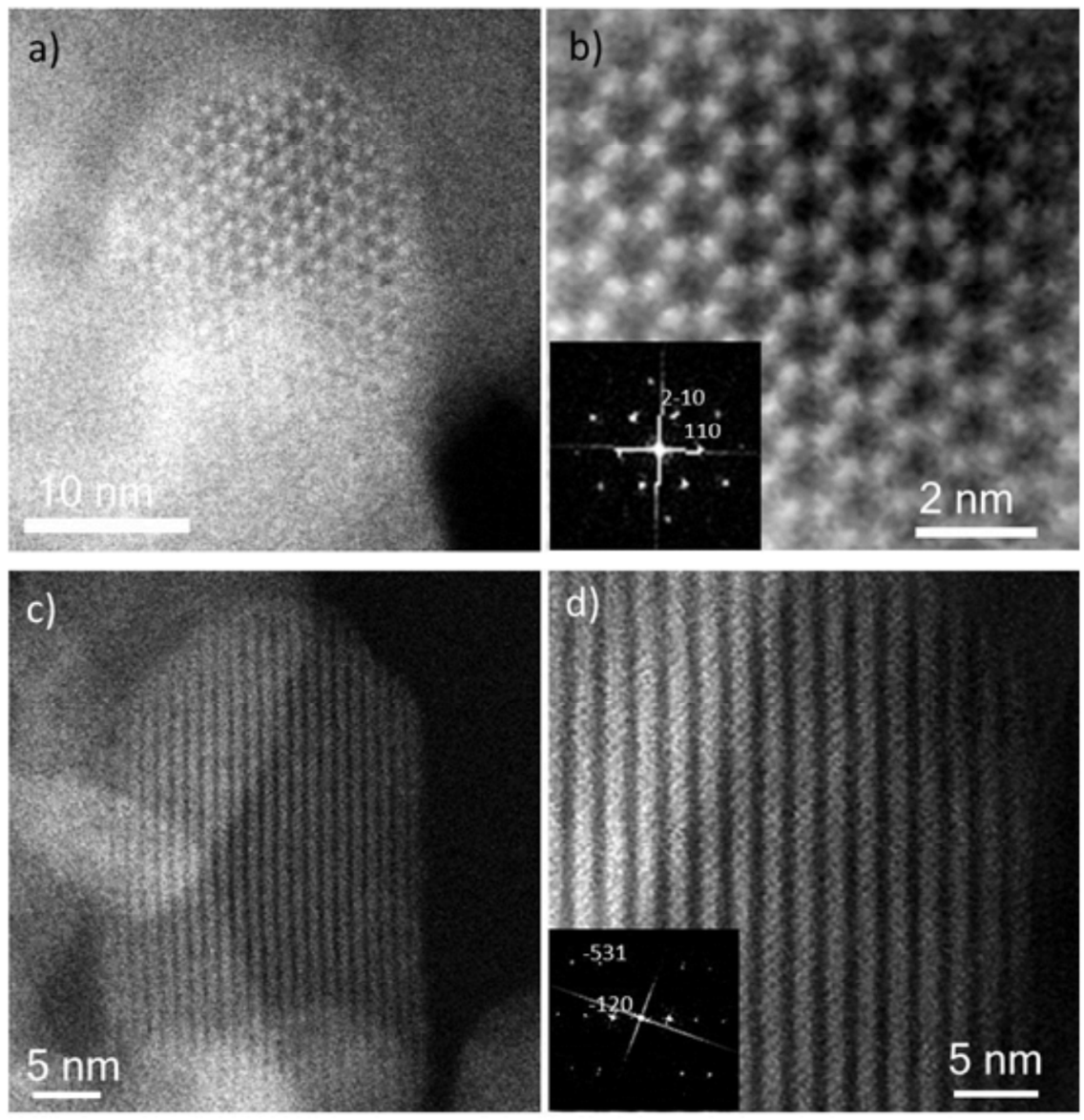

Figure 8. a) Cs corrected STEM-HAADF images of a nanoparticle faced along the [001] zone axis. b) Fourier filtered image of a) displaying the pore architecture, with FFT inset. c) A different crystal with the pore system perpendicular to the electron beam. d) A filtered Fourier region of c) with the FFT inset indexed assuming the [211] orientation.

materials. ${ }^{44}$ Taking into account the extremely high instability of MOFs under the electron beam, only few studies have been reported on high-resolution TEM by using a cryo TEM technique. ${ }^{36}$, ${ }^{45}$ However, in the current case a different approach based on the results already produced for zeolites has been taken. Figures 8a-d present extremely high-resolution data obtained for two different Zn-MOF-74 crystals. (Some other so-obtained high-resolution images of a different crystal of the same sample is shown in Figure S16). Figure 8a shows the raw data of a $20 \mathrm{~nm}$ crystal orientated along the [001] direction exhibiting a hexagonal arrangement of the pores. Figure $8 \mathrm{~b}$ presents a closer look at the pores of the particle, where in bright, due the stronger 
scattering factor, the Zn cluster can be unambiguously identified. In addition the FFT, displaying a 6-fold axis shown inset, which corroborates the high degree of crystallinity of each individual entity, was indexed in the $R-3 m$ space group with $\mathrm{a}=\mathrm{b}=25.93 \AA$ and $\mathrm{c}=6.83 \AA$. Figures $8 \mathrm{c}$ and 8d (raw data and Fourier filtered image respectively) display the image of another Zn-MOF-74 particle sitting with the channels perpendicular to the electron beam. To the best of our knowledge, this is the very first time that such a high-resolution data is produced from a MOF system which allows the observation of the metal cluster linking the organic phase. Such image allows a direct measurements of the distance between two diagonal $\mathrm{Zn}$ units being $15.5 \AA$, leading into a pore size of approximately $10.54 \AA .^{46}$ The fact that individual atomic Zn columns has not been resolved as it has been done in other porous architectures ${ }^{47}$ is associated to the weakness of the Zn-MOF-74 framework which forced to work under very far-from-ideal fast conditions in order to minimize the beam damage.

\section{DISCUSSION}

The different characterization techniques used in this work have provided very different and valuable insights into the size of both crystals and void spaces enclosed by crystal agglomeration / aggregation of the series of M-MOF-74 materials. At first glance, not all these insights are in good agreement. In this section, all these estimations are discussed and considered together.

According to the estimation through Scherrer equation, Ni and Co samples are formed by crystal diameters as small as 2.8 and $5.1 \mathrm{~nm}$, respectively. They are probably the smallest crystal size of a microporous material ever reported. To the best of our knowledge, nano-sized Beta zeolite crystals had that record with $c a .7 \mathrm{~nm}$ of crystal diameter when it has been prepared by very different approaches ${ }^{43,48}$. The XRD patterns of those beta zeolite samples obviously had 
broad peaks but they did not reach the magnitude of those of our samples. Moreover, one particular dimension of MFI-structured crystals has been reduced practically to a single unit cell $^{49,50}$. Taking into account that the dimensions of a unit cell of $\mathrm{Zn}-\mathrm{MOF}-74$ are of $\mathrm{a}=\mathrm{b}=2.593$ $\mathrm{nm}, \mathrm{c}=0.684 \mathrm{~nm}^{46}$, a $c$-axis transversal section of an averaged crystal of Ni-MOF-74 $(2.8 \mathrm{~nm})$ and Co-MOF-74 $(5.1 \mathrm{~nm})$ materials contains slightly more than a single (1.15 unit cells) and four unit cells, respectively, justifying their almost negligible diffraction capacity (Figure 1) and their lower micropore textural properties (Table S1) in comparison with their homologues composed by micrometer crystals. We should not reject the possibility that the crystals are even smaller, as for instance discrete domains of size smaller than a unit cell could potentially exist but do not significantly contribute to the XRD detected peaks, so the estimated size would be that only averages the size of the crystals able to diffract at some non-null extent.

Unlike the conventional M-MOF-74 materials, the formation of these nano-sized MOFs takes place through a precipitation mechanism. Since the fundaments of precipitation process are wellknown, we have tried to correlate the estimated crystal size of M-MOF-74 materials with different physic-chemical properties conditioning the formation of a precipitate. One of the most influent parameters is the solubility of the chemical species taking part in the final precipitate. Indeed, M-MOF-74 cannot be formed at room temperature if $\mathrm{M}$ is not added as acetate but as nitrate or chloride, which are the conventional sources for solvothermal synthesis methods. Figure 9 plots the solubility of the used M acetates in water as a function of crystal width of the resultant M-MOF-74 material estimated by Scherrer equation. We are aware that the major solvent in our system is N,N-dimethylformamide (DMF), so the use of tabulated values of solubility in water ${ }^{51}$ is not strictly correct. However, it is expected that the order of solubility of ionic salts in so polar solvent as water and DMF is the same. Figure 9 shows an almost linear 
correlation between so-represented solubility and crystal width when $\mathrm{Mn}, \mathrm{Mg}, \mathrm{Co}$ and $\mathrm{Ni}$ acetates (but not $\mathrm{Zn}$ ) one are considered. The reason behind the mismatch of $\mathrm{Zn}$ in that correlation could be the different hydration degree of its source (two water molecules per $\mathrm{Zn}$ ) in comparison with the rest $\mathrm{M}$ acetates (4 water molecules per $\mathrm{M}$ ), considering the strong capacity of water to dissolve any of these acetates. The good correlation between solubility and crystal size among the comparable metal acetates, opens the possibility of accurately controlling the crystal size of M-MOF-74 materials (and indirectly their agglomeration or aggregation features) by just changing the solubility of the metal sources, either modifying the counter-ion nature or the solvent mixture nature / proportion.

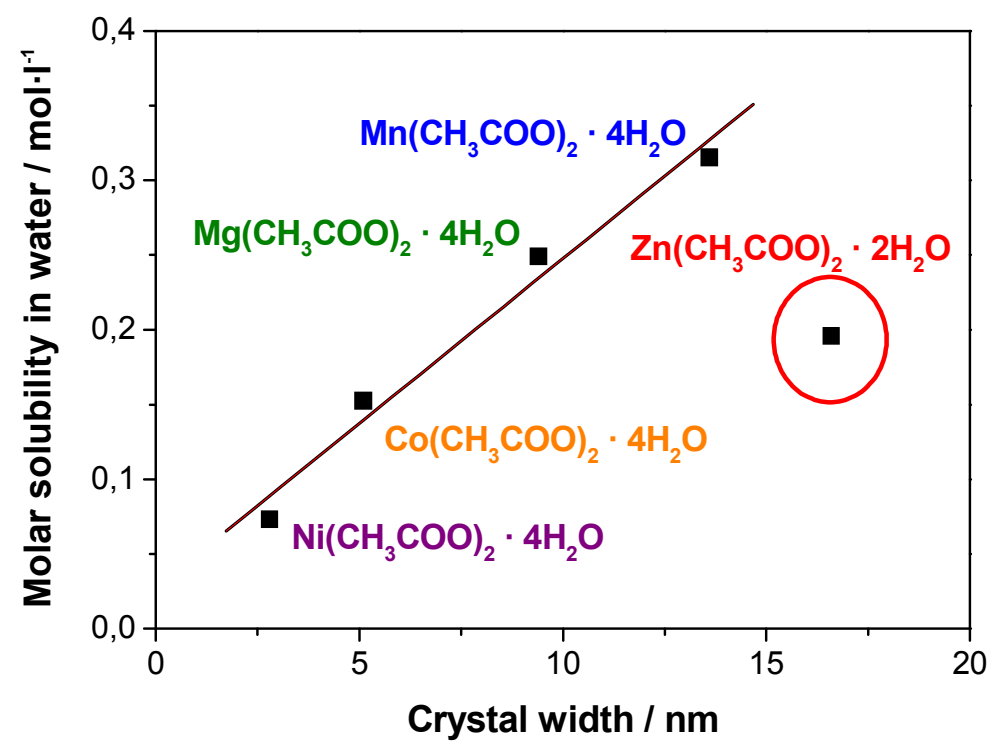

Figure 9. Molar solubility in water of the different metal acetates sources ${ }^{51}$ used in this work versus crystal width of the corresponding M-MOF-74 materials estimated from Scherrer equation. The black line represents the straight line better fitted to the tetrahydrate acetate sources. Red circle is used to remark that $\mathrm{Zn}$ does not follow such trend.

Figure 10 shows the evolution of the some data estimated or determined by different characterization techniques used in this study as a function of the nature of the divalent metal M in the series of the M-MOF-74 samples. In general terms, all three plotted magnitudes have almost systematically decreased in the order of $\mathrm{M}: \mathrm{Zn}>\mathrm{Mn}>\mathrm{Mg}>\mathrm{Co}>\mathrm{Ni}$. The unique non- 
systematic slight variation comes from the low-angle peak, whose origin can be influenced by the crystal morphology, crystal size homogeneity, certain spatial ordering of either nanoparticles or mesopores or even a mixture of them. In any case, this magnitude also follows the general trend defined by the other two plotted magnitudes, whose origin is more well-defined and easily interpretable. A second significant feature from Figure 9 is that the practically linear evolution of crystal size estimated by Scherrer equation along the $\mathrm{M}$ series contrasts with the abrupt fall and higher range found in PSD maxima evolution, coinciding with the vertical dashed line. The higher value of PSD maxima than the width found in the two samples with largest nanocrystals ( $\mathrm{Zn}$ and $\mathrm{Mn}$ ) can be explained based on the fact that as-applied Scherrer equation provides the width (the smallest dimension) of the rod-like nanocrystals. Consequently, it is not surprising that the mesopores formed by agglomeration of that nanocrystals are larger than the width of those crystals. This effect is visually made clear in Figure 6-right and Figures S14 and S17 of Supporting Information. As the nanocrystals become smaller, they are larger than the pore defined by them. It could be due to a more strong interaction between the nanoparticles, provoking that the presumably more reactive nanocrystals somehow loss their identity becoming simple domains in hardly-dissociable particles (Figure 6-left, Figures S18-S23 of Supporting Information). Mg-MOF-74 is an acceptable midpoint between the two scenarios, as some discrete units can be intuited although it is dominated by 'fused' crystals (Figure S16C). As a consequence, around $10 \mathrm{~nm}$ of crystal/domain size would mark a different behavior of nanocrystalline M-MOF-74 materials (vertical dashed line in Figure 9). Above that size, it is properly to talk about more or less separable nanocrystals, whereas below, crystalline domains exist. 
Therefore, estimating / measuring the crystal / domain size are not enough to design potential application of these materials. Additionally, it is also recommendable to have a deep knowledge of the nanocrystals agglomeration / aggregation / fusion process. As illustrative examples, we can anticipate two different situations for the two applications mentioned in the Introduction section as improvable by using nano-sized MOFs crystals. On one hand, the use of these MOFs as catalyst would potentially reduce / resolve the diffusional problems of their micrometer-size homologues. On the other hand, their use as fillers in polymer-based membranes to give high homogeneity at 'nano' scale would be presumably reached just in the case of the samples formed by separable over-10-nm crystals, whereas samples formed by large particles of nanoscopic domains could be even counterproductive.

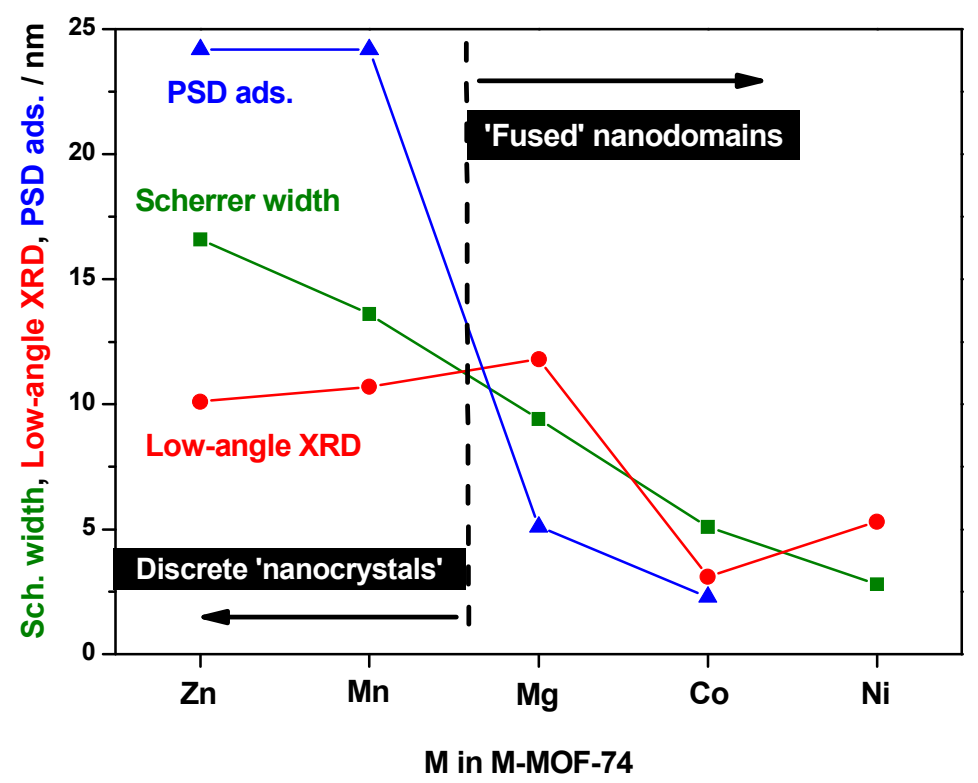

Figure 10. Comparison of crystal widths as estimated by Scherrer equation from PXRD patterns (green squares), position of the low-angle XRD peak (red circles) and pore size distribution maxima from the $\mathrm{N}_{2}$ adsorption isotherms (blue triangles) of the nanocrystalline M-MOF-74 samples as a function of the nature of $\mathrm{M}$. The vertical dashed line and the regions marked in the Figure are interpreted in the text. 


\section{CONCLUSIONS}

Some important applications of MOFs could be enhanced by decreasing their crystal size down to the nano-scale. This work describes an easy, quick and economy preparation of a set of nanocrystalline M-MOF-74 materials ( $\mathrm{M}=\mathrm{Zn}, \mathrm{Mn}, \mathrm{Mg}$, Co and Ni). The resultant materials are composed by so small crystals that their identification by diffraction techniques is flagrantly insufficient. Indeed, the Co- and Ni-MOF-74 samples are probably the MOFs materials composed by smallest crystals ever reported. A combination of characterization techniques corroborates both their 'nano' condition and their crystalline nature of MOF-74 materials. Additionally, all samples contain some textural porosity of nature and magnitude well-explained from their crystal size and the tendency of their nanocrystals to be agglomerated or aggregated. Zn-MOF-74 nanocrystals could be isolated by relatively soft ultrasonic treatment; indeed, these isolated crystals could be observed by $\mathrm{C}_{\mathrm{s}}$-corrected STEM with an unprecedented resolution by any microscopy technique, as the metal clusters and the hexagonal channel of $11.5 \AA$ of diameter were sharply observed. On the contrary, Co and Ni samples are formed by nanodomains 'fused' in large particles rather than by discrete nanocrystals. Consequently, isolated domains could not be separated by conventional ultrasonic treatments. Moreover, the control of the crystal size has been suggested to be a function of solubility of the metal source in the synthesis media, and then it could be controlled. The described behaviour of the different degree and/or nature of nanocrystals / nanodomains aggregation as a function of crystal size could be crucial in the design of new MOFs and, more importantly, in the MOFs applications favoured by reduced crystal size. 


\section{ASSOCIATED CONTENT}

Supporting Information. Further XRD analyses (influence of fluorescence, low-angle PXRD, Thermo-XRD, kinetics of formation followed by XRD), further TG analyses (quantification, effect of nanosized on thermal stability), further $\mathrm{N}_{2}$ adsorption/desorption isotherm analyses (PSD from desorption branch), further electron microscopy images and dynamic light scattering (DLS) measurements.

\section{AUTHOR INFORMATION}

Corresponding Author

* Phone: +34-915854795; Fax:+34-915854760. E-mail address: manuel.sanchez@icp.csic.es

\section{Funding Sources}

Spanish Ministry (MAT-2012-31127) and European Union Seventh Framework Programme under Grant Agreement 312483 - ESTEEM2 (Integrated Infrastructure Initiative-I3).

\section{ACKNOWLEDGMENT}

We are very grateful to the technicians forming the 'Unidad de Apoyo a la Investigación' of ICP, particularly to Conchi Díaz and Rosa Folgado, for their unselfish and intense involvement in this work.

\section{REFERENCES}

(1) Li, H.; Eddaoudi, M.; O'Keeffe, M.; Yaghi, O. M., Nature 1999, 402, (6759), 276-279.

(2) Issue 5 Chem. Soc. Rev. 2009, 38, (5), 1201-1508. 
(3) Issue 2 Chem. Rev. 2012, 112, (2), 673-1268.

(4) Kuppler, R. J.; Timmons, D. J.; Fang, Q.-R.; Li, J.-R.; Makal, T. A.; Young, M. D.; Yuan, D.; Zhao, D.; Zhuang, W.; Zhou, H.-C., Coord. Chem. Rev. 2009, 253, (23-24), 3042-3066.

(5) O'Keeffe, M.; Peskov, M. A.; Ramsden, S. J.; Yaghi, O. M., Acc. Chem. Res. 2008, 41, 1782-1789.

(6) Bell, A. T., Science 2003, 299, (5613), 1688-1691.

(7) Egeblad, K.; Christensen, C. H.; Kustova, M.; Christensen, C. H., Chem. Mater. 2008, 20, 946-960.

(8) Oh, M.; Mirkin, C. A., Nature 2005, 438, (7068), 651-654.

(9) Horcajada, P.; Serre, C.; Grosso, D.; Boissiere, C.; Perruchas, S.; Sanchez, C.; Ferey, G., Adv. Mater. (Weinheim, Ger.) 2009, 21, (19), 1931-1935.

(10) Spokoyny, A. M.; Kim, D.; Sumrein, A.; Mirkin, C. A., Chem. Soc. Rev. 2009, 38, (5), 1218-1227.

(11) Shekhah, O.; Liu, J.; Fischer, R. A.; Woll, C., Chem. Soc. Rev. 2011, 40, (2), 1081-1106.

(12) Ma, M.; Zacher, D.; Zhang, X.; Fischer, R.A.; Metzler-Nolte, N. Cryst. Growth Des. 2011, 11, 185-189.

(13) Botella, P.; Corma, A.; Lopez-Nieto, J. M.; Valencia, S.; Jacquot, R., J. Catal. 2000, 195, (1), 161-168.

(14) Dhakshinamoorthy, A.; Alvaro, M.; Hwang, Y. K.; Seo, Y. K.; Corma, A.; Garcia, H., Dalton Trans. 2011, 40, (40), 10719-10724.

(15) Zornoza, B.; Gorgojo, P.; Casado, C.; Tellez, C.; Coronas, J., Desalin. Water Treat. 2011, 27, (1-3), 42-47.

(16) Caro, J., Current Opinion in Chemical Engineering 2011, 1, (1), 77-83.

(17) Cundy, C. S.; Cox, P. A., Chem. Rev. 2003, 103, 663.

(18) Stock, N.; Biswas, S., Chem Rev 2012, 112, (2), 933-69.

(19) Friščić, T., J. Mater. Chem. 2010, 20, (36), 7599.

(20) Son, W. J.; Kim, J.; Kim, J.; Ahn, W. S., Chem Commun (Camb) 2008, (47), 6336-8.

(21) Klinowski, J.; Paz, F. A.; Silva, P.; Rocha, J., Dalton Trans. 2011, 40, (2), 321-30.

(22) Kaye, S. S.; Dailly, A.; Yaghi, O. M.; Long, J. R., J. Am. Chem. Soc. 2007, (129), 14176.

(23) Calleja, G.; Botas, J. A.; Orcajo, M. G.; Sanchez-Sanchez, M., J. Porous Mater. 2010, 17, (1), 91-97.

(24) Tranchemontagne, D. J.; Hunt, J. R.; Yaghi, O. M., Tetrahedron 2008, 64, (36), 85538557.

(25) Millward, A. R.; Yaghi, O. M., J. Am. Chem. Soc. 2005, 127, (51), 17998-9.

(26) Dietzel, P. D.; Panella, B.; Hirscher, M.; Blom, R.; Fjellvag, H., Chem. Commun. (Cambridge, U. K.) 2006, (9), 959-61.

(27) Caskey, S. R.; Wong-Foy, A. G.; Matzger, a. A. J., J. Am. Chem. Soc. 2008, 130, 10870.

(28) Dietzel, P. D.; Georgiev, P. A.; Eckert, J.; Blom, R.; Strassle, T.; Unruh, T., Chem Commun (Camb) 2010, 46, (27), 4962-4.

(29) Botas, J. A.; Calleja, G.; Sanchez-Sanchez, M.; Orcajo, M. G., Int. J. Hydrogen Energy 2011, 36, (17), 10834-10844.

(30) Schoenecker, P. M.; Carson, C. G.; Jasuja, H.; Flemming, C. J. J.; Walton, K. S., Ind. Eng. Chem. Res. 2012, 51, (18), 6513-6519.

(31) Sillar, K.; Sauer, J., J. Am. Chem. Soc. 2012, 134, (44), 18354-65.

(32) Dietzel, P. D. C.; Blom, R.; Fjellvag, H., Eur. J. Inorg. Chem. 2008, (23), 3624-3632. 
(33) Sumida, K.; Rogow, D. L.; Mason, J. A.; McDonald, T. M.; Bloch, E. D.; Herm, Z. R.; Bae, T. H.; Long, J. R., Chem Rev 2012, 112, (2), 724-81.

(34) Sumida, K.; Brown, C. M.; Herm, Z. R.; Chavan, S.; Bordiga, S.; Long, J. R., Chem. Commun. (Cambridge, U. K.) 2011, 47, (4), 1157-1159.

(35) Suh, M. P.; Park, H. J.; Prasad, T. K.; Lim, D. W., Chem Rev 2012, 112, (2), 782-835.

(36) Deng, H.; Grunder, S.; Cordova, K. E.; Valente, C.; Furukawa, H.; Hmadeh, M.; Gandara, F.; Whalley, A. C.; Liu, Z.; Asahina, S.; Kazumori, H.; O'Keeffe, M.; Terasaki, O.; Stoddart, J. F.; Yaghi, O. M., Science 2012, 336, (6084), 1018-23.

(37) Burton, A. W.; Ong, K.; Rea, T.; Chan, I. Y., Microporous Mesoporous Mater. 2009, 117, (1-2), 75-90.

(38) Manjón-Sanz, A.; Sánchez-Sánchez, M.; Muñoz-Gómez, P.; García, R.; Sastre, E., Microporous Mesoporous Mater. 2010, 131, (1-3), 331-341.

(39) Olivares, M.; Larranaga, A.; Irazola, M.; Sarmiento, A.; Murelaga, X.; Etxebarria, N., Talanta 2012, 98, 172-177.

(40) FitzGerald, S. A.; Burkholder, B.; Friedman, M.; Hopkins, J. B.; Pierce, C. J.; Schloss, J. M.; Thompson, B.; Rowsell, J. L., J. Am. Chem. Soc. 2011, 133, (50), 20310-8.

(41) Sing, K. S. W.; Everett, D. H.; Haul, R.A.W.; Moscou, L.; Pierotti, R. A.; Rouquerol, J.; Siemieniewska, T., Pure Appl. Chem. 1985, 57, (4), 603-618.

(42) Sergej, N. Hysteresis Phenomena in Mesoporous Materials. Leipzig University, 2009.

(43) Camblor, M. A.; Corma, A.; Valencia, S., Microporous Mesoporous Mater. 1998, 25, 5974.

(44) Mayoral, A.; Coronas, J.; Casado, C.; Tellez, C.; Díaz, I., ChemCatChem 2013, n/a-n/a.

(45) Wiktor, C.; Turner, S.; Zacher, D.; Fischer, R. A.; Van Tendeloo, G., Microporous Mesoporous Mater. 2012, 162, 131-135.

(46) Rosi, N. L.; Kim, J.; Eddaoudi, M.; Chen, B. L.; O'Keeffe, M.; Yaghi, O. M., J. Am. Chem. Soc. 2005, 127, (5), 1504-1518.

(47) Mayoral, A.; Carey, T.; Anderson, P. A.; Lubk, A.; Diaz, I., Angew. Chem.-Int. Edit. 2011, 50, (47), 11230-11233.

(48) Schmidt, I.; Madsen, C.; Jacobsen, C. J. H., Inorg. Chem. 2000, 39, 2279-2283.

(49) Choi, M.; Na, K.; Kim, J.; Sakamoto, Y.; Terasaki, O.; Ryoo, R., Nature 2009, 461, (7261), 246-9.

(50) Zhang, X.; Liu, D.; Xu, D.; Asahina, S.; Cychosz, K. A.; Agrawal, K. V.; Al Wahedi, Y.; Bhan, A.; Al Hashimi, S.; Terasaki, O.; Thommes, M.; Tsapatsis, M., Science 2012, 336, (6089), 1684-7.

(51) http://www.chemnet.com/ http://www.chemnet.com/. 


\section{For Table of Contents Use Only}

Nano-scaled M-MOF-74/CPO-27-M (M= Mg, Mn, Co, Ni and Zn) materials has been at room temperature. The crystals/domains are in the limit of being able to diffract, particularly those forming the Co- and Ni-MOF-74 samples. Zn-MOF-74 nanocrystals are imaged by spherical aberration (Cs) corrected STEM techniques, showing unprecedented 'quasi' atomic resolution electronic microscopy images of a MOF material.

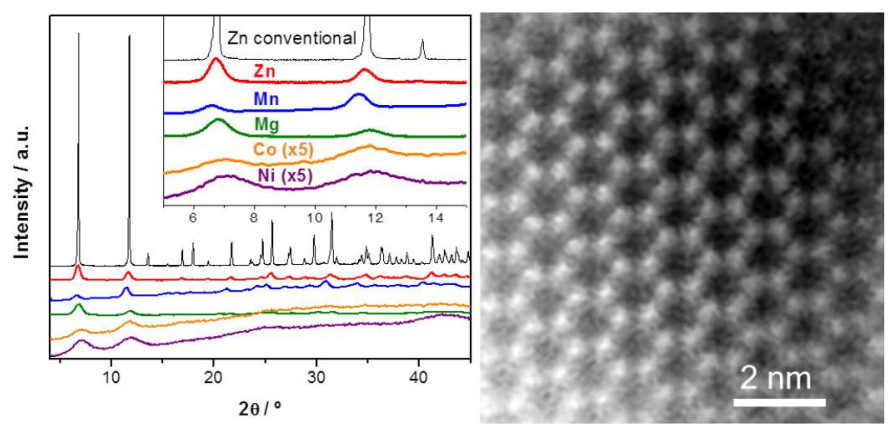

